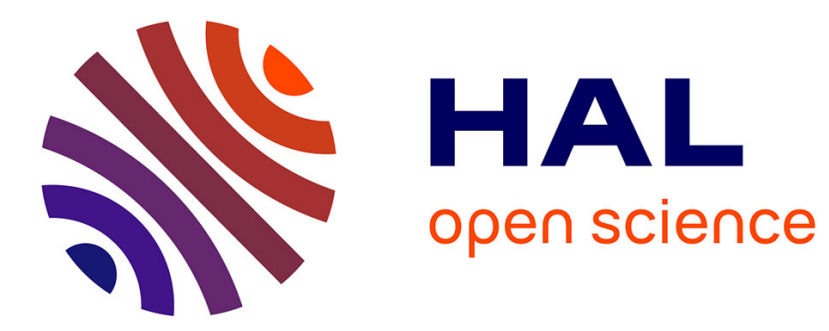

\title{
L'intonation des musiques monodiques: méthodologies expérimentales
}

E. James (wasiuddin) Arnold, Bernard Bel

\section{To cite this version:}

E. James (wasiuddin) Arnold, Bernard Bel. L'intonation des musiques monodiques: méthodologies expérimentales. Revue de musicologie, 1985, T. 71e (1-2), pp.159-163. hal-00370250

\section{HAL Id: hal-00370250 \\ https://hal.science/hal-00370250}

Submitted on 24 Mar 2009

HAL is a multi-disciplinary open access archive for the deposit and dissemination of scientific research documents, whether they are published or not. The documents may come from teaching and research institutions in France or abroad, or from public or private research centers.
L'archive ouverte pluridisciplinaire HAL, est destinée au dépôt et à la diffusion de documents scientifiques de niveau recherche, publiés ou non, émanant des établissements d'enseignement et de recherche français ou étrangers, des laboratoires publics ou privés. 


\title{
L'intonation des musiques monodiques : méthodologies expérimentales
}

\author{
E. James ARNOLD, Bernard BEL \\ International Society for Traditional Arts Research (ISTAR)
}

Rédigé en 1980. Publié dans la Revue de Musicologie, vol. T. 71e, no. 1-2. 1985, p. 159-163

Bien que l'étude expérimentale de l'intonation de la musique classique de l'Inde ait fait l'objet de diverses tentatives depuis le début de ce siècle, les difficultés d'une telle entreprise ne peuvent être surmontée qu'avec les technologies et les méthodes mathématiques les plus récentes.

Les approches qui se sont inspirées de travaux théoriques antérieurs, comme par exemple ceux de Clements et Deval ${ }^{1}$ ou de Daniélou ${ }^{2}$ (1968) ont abouti à "prouver » ces théories contradictoires. Plus récemment, les études de Jairazbboy ${ }^{3}$ et de son élève Levy $^{4}$ tendent à déprécier l'importance du sujet en utilisant des méthodologies qui conduisent naturellement à cette conclusion.

Il est intéressant de noter que les musicologues qui ont étudié le problème à partir d'une formulation numérique du système d'intonation juste (Clements, Daniélou, Modak ${ }^{5} .$. ) ont construit des instruments à clavier ou à cordes (Shruti harmonium, Shruti vina) destinés à démontrer les avantages de ce système dans la pratique musicale. Nous avons recueilli des membres de la famille Dagar le témoignage d'une rencontre historique entre leurs aïeuls Allahbande Khan et Zakiruddin Khan, et Clements et Deval au début de ce siècle. Ayant tenté de démontrer les qualités du Shruti harmonium comme instrument d'accompagnement devant une large audience, ces derniers ont dû s'incliner devant l'évidence que les positions tonales de leur instrument n'étaient pas celles utilisées par ces deux grands experts de l'intonation! Nous avons nous-mêmes été placés dans une situation analogue face à leur petit-fils Rahim Fahimuddin Dagar...

Il n'en reste pas moins que de nombreux musiciens ont une idée très précise des positions tonales à utiliser dans un raga et dans un contexte mélodique définis, et qu'ils sont capables même de manipuler ces positions dans une fourchette de quelques schismas ( $1 / 2$ savart ou 2 cents).

Plus récemment, les travaux de Jairazbhoy et de Levy, fondés sur la mesure de fréquence de certaines notes sur un enregistrement avec une précision de plus ou moins deux schismas ${ }^{6}$, ont montré qu'il existe des déviations de positions tonales

${ }^{1}$ E. Clements, Introduction to the Study of Indian Music (Allahabad Kitab Mahal, 1912) ; K. B. Deval, Music East and West Compared (Poona : Ayabhushan Press, 1908).

${ }^{2}$ A. Daniélou, Introduction to the Study as Musical Scales (London : The India Socie ty, 1943). The ragas of North Indian music (London Barrie \& Rockliff, 1968). Ces deux ouvrages ont été réimprimés en Inde.

${ }^{3}$ N.A. Jairazbhoy e t A.W. Stone. « Intonation in Present -Day North Indian Classical Music ». Bulletin of the School of Oriental and African Studies, XXVI (1963).

${ }^{4}$ M. Levy, Intonation in North Indian Music (New Dehli : Biblia Impex, 1982).

${ }^{5}$ H.V. Modak, S.R. Chandarkar, S. Parameswaran et B.K. Parikh, «Study of Musical Scales in Practise », Report of Symposium New Delhi (Sangeet Natak Akademi, 1975).

${ }^{6}$ M. Levy, op. cit., p. 93. 
dans une fourchette de plus ou moins 7 savarts (30 cents) autour de la position tempérée pour certaines notes. Ce qui les a conduits à conclure que "l'intonation juste n'est pas un sujet de grande importance dans la musique du nord de l'Inde... Il apparaît que l'intonation est une question de choix personnel... $»^{7}$ et que $« \ldots$ la gamme tempérée occidentale se situe dans l'intervalle de tolérance des diverses notes des gammes utilisées couramment dans la musique classique du nord de $1^{\prime}$ Inde. $»^{8}$ Conclusions qui soulèvent un tollé général parmi les musiciens traditionnels !

L'analyse acoustique des sons musicaux est certes une science, mais son objet est un matériau fait de "main » d'hommes; c'est pourquoi on ne peut ignorer sa «subjectivité ». Travaillant sur des enregistrements de R. Fahimuddin Dagar, nous avons eu l'occasion de lui demander son avis sur certaines notes qui avaient attiré notre attention par leur position particulière. Ces positions furent souvent identifiées comme des erreurs. Il peut être intéressant d'analyser les erreurs de musiciens aussi bien que leurs notes justes - à condition de savoir lesquelles sont justes ou non - mais on ne peut que mettre en doute la valeur des études basées sur des enregistrements de maîtres disparus depuis vingt ans.

\section{Le Shruti harmonium SH 1000}

Nous utilisons, depuis janvier 1980, dans le cadre de notre projet «Étude scientifique de la musique modale du nord de l'Inde » entrepris sous l'égide du Fonds International pour le Promotion de la Culture (UNESCO), un Shruti Harmonium programmable électronique. Cet orgue polyphonique possède un clavier standard dodécaphonique de quatre octaves, dont les touches peuvent être accordées avec une précision meilleure que $1 / 1000$ (1/3 de savart, ou 5/6 de cent). Les rapports de fréquence (le Do servant de référence) sont programmés à partir d'un clavier numérique et stockés dans une mémoire non volatile.

Chaque touche a accès à quatre positions pré-programmées grace à un jeu d'interrupteurs en face avant. L'accord général de l'instrument peut être déplacé sur une octave, avec une définition de 1/2000, sans affecter les intervalles. Avec ce type d'instrument, il est donc possible de programmer facilement n'importe quel système de division de l'octave à partir de données théoriques, puis de modifier la programmation en fonction des résultats auditifs.

Cet instrument, conçu et réalisé par les auteurs, comporte d'autre part un jeu de douze filtres programmables de conception nouvelle et dont l'effet sélectif est comparable à celui des cordes sympathiques du sitar ou du sarangi (tarab électronique). Ces filtres répondent lorsque les positions tonales utilisées par le musicien coïncident avec celles programmées sur le clavier.

Bien que cet instrument ait été accueilli très favorablement par les musicologues et les musiciens de l'Inde, nous avons pris conscience de ses limitations pour ce type de recherche. Les exigences des musiciens, que cet instrument ne peut satisfaire, mettent en évidence certains phénomènes d'importance capitale pour ce qui est de la nature même des sons musicaux. En fait, l' «intonation» de la musique de l'Inde est moins une question de "notes justes" isolées que de "micro-évolution » de la ligne mélodique : non seulement il faut prendre en compte les glissements mélodiques bien connus (entre deux notes, par exemple), mais aussi les ondulations très subtiles (dans une fourchette de quelques schismas, ou même les changements de timbre ou de voyelle) sur une position plus ou moins stable...

D'autre part les phénomènes d'aberration de perception de hauteur (fonctions de l'intensité, du timbre, ou de l'inharmonicité des partiels) bien connus dans le

${ }^{7}$ N.A. Jairazbhoy, op. cit., p. 130-131.

${ }^{8}$ N.A. Jairazbhoy, op. cit., p. 129 ; cité par Levy, op. cit., p. 73. 
contexte de sons isolés, interviennent aussi en présence du tanpura (instrument à cordes donnant un bourdon continu). Il importe donc de ne pas confondre les notions de «hauteur» (perçue) et de «fréquence» (mesurée). Il en découle que l'étude de la ligne mélodique doit prendre en compte de nombreux paramètres qui permettent ultérieurement de replacer tout phénomène dans son contexte.

\section{L'analyseur de mouvement mélodique MMA1}

Les considérations qui précèdent nous ont amenés à réaliser un analyseur de type nouveau, s'adaptant modulairement à diverses situations expérimentales (par le choix des paramètres enregistrés) tout en utilisant de manière optimale les performances du système de stockage des mesures. Les mesures sont converties en données numériques et stockées à la cadence de 10000 bits par seconde sur une piste de la bande magnétique, tandis que le son musical est préservé sur l'autre piste.

La figure 1 illustre un exemple d'enregistrement simultané de paramètres acoustiques et de paramètres psycho-physiologiques. La fréquence d'échantillonage des paramètres, fonction du nombre d'entrées utilisées (ou, plus précisément, de «bits »), est ici de 70 cycles par seconde. La fréquence du fondamental de la ligne mélodique et celle du tanpura sont mesurées avec une précision meilleure que 1/4000 (1/12 de savart ou 1/4 de cent). Vingt-sept filtres passe-bande mesurent la répartition de l'énergie sonore dans une partie du spectre, pour l'analyse des voyelles chantées. Certains de ces filtres peuvent aussi être affectés au tanpura. Les paramètres psycho-physiologiques ne sont cités qu'à titre d'exemple. Nous étudions actuellement (juillet 1982) la possibilité de réaliser en temps réel l'analyse spectrale des ondes cérébrales (EEG) pour les parties droite et gauche du cerveau, dispositif qui fournirait des informations intéressantes sur l'activité psychique d'un auditeur.

La figure 2 montre comment les mesures sont traitées. Les passages à analyser sont repérés sur la piste sonore, et les données correspondantes sont transférées dans la mémoire de l'ordinateur, puis sur un disque souple (floppy disk). Les modes de traitement sur lesquels nous portons notre attention en premier lieu sont les suivants :

1. Analyse de la ligne mélodique et détermination statistique des positions tonales utilisées par un musicien dans un contexte donné ;

2. Corrélation entre les formes des voyelles, l'enveloppe du son, et les « micro-ajustements » de positions tonales (phénomènes de distorsion de la perception de hauteur) ;

3. Intégration de la ligne fréquentielle en vue de déterminer les «poids » des notes et leur fonction dans une phrase mélodique, ou un raga donnée ${ }^{9}$;

4. Étude morphologique des formes d'ornementation (alankar);

5. Corrélation des paramètres acoustiques, de l'ambiance (rasa) du raga, et des paramètres psychophysiologiques.

${ }^{9}$ Voir E. Leipp, La Machine à écouler (Paris: Massori, 1977), p. 182. 


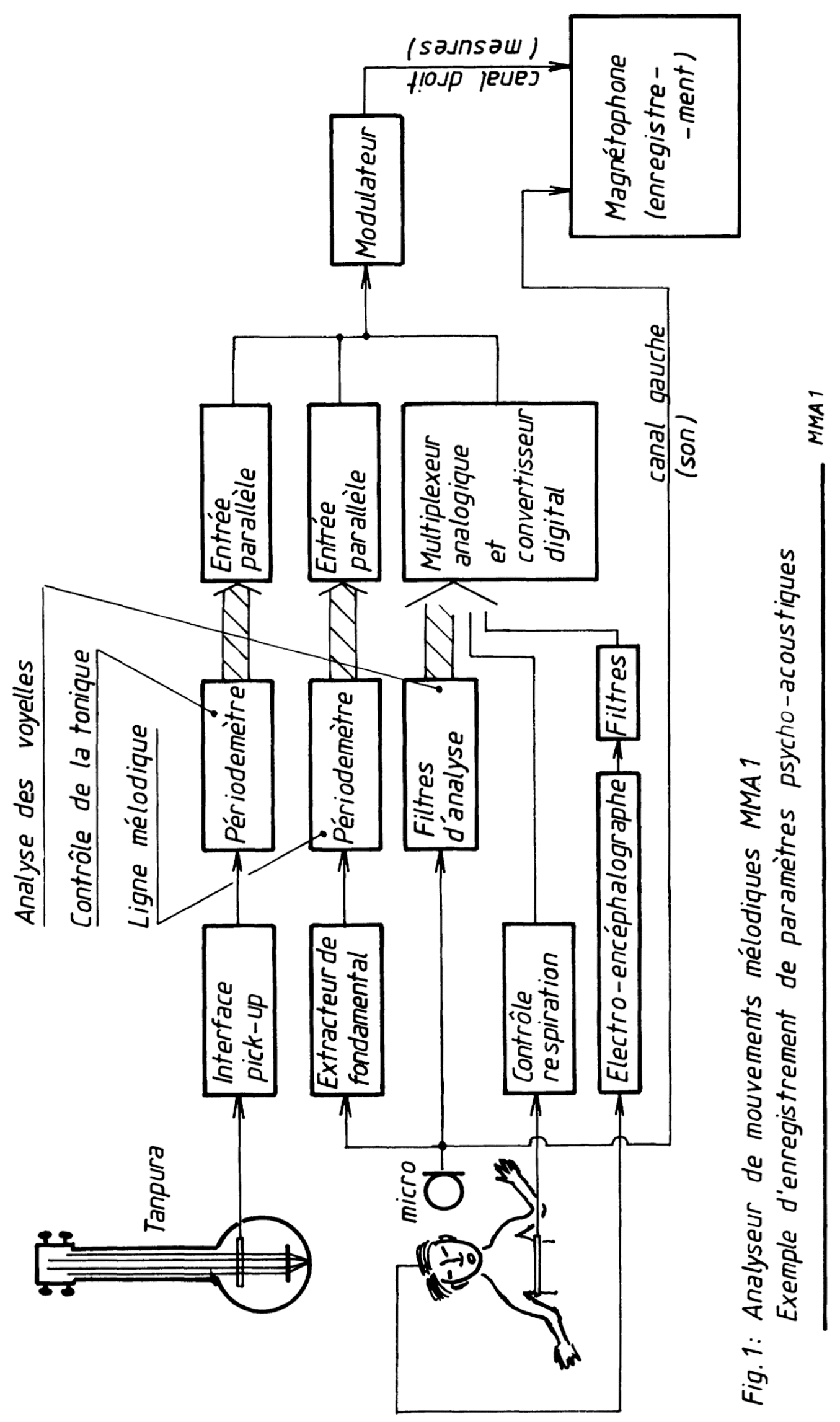




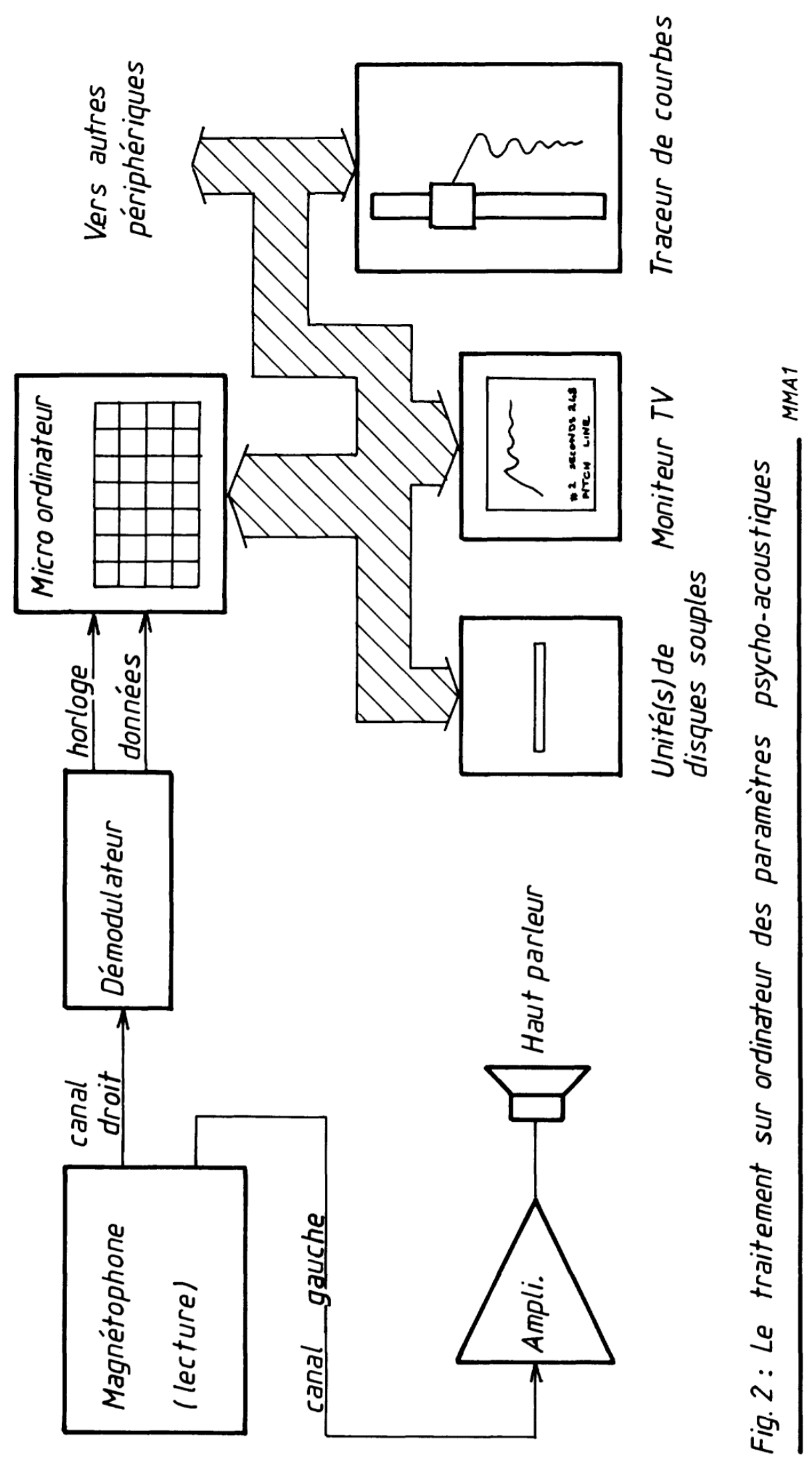

